American Journal of Nanotechnology 1 (2): 51-55, 2010

ISSN 1949-0216

(C) 2010 Science Publications

\title{
Development of a Soft-Computer Numerical Control System for Micro-Electrochemical Machining
}

\author{
Yukui Wang, Hui Chen, Zhenlong Wang, Zhiwei Zhou and Debin Shan \\ Mechanical Engineering, \\ Materials Science and Engineering, Harbin Institute of Technology, Harbin, China
}

\begin{abstract}
Problem statement: In this study a kind of soft Computer Numerical Control (soft-CNC) system for micro Electrochemical Machining (micro-ECM) based on RT-Linux platform is developed. Approach: Dual-kernel structure of System is adopted: Linux and RT-Linux are used to realize timesharing tasks and real-time tasks separately. Results: The CNC Graphical User Interface (GUI) is designed by Qt and runs on Linux core space. Based on the hard real-time facilities provided by RTLinux, the real-time tasks, including the gap detection task, gap adjustment task, interpolating task and other real-time control tasks are implemented. The Shared Memory (SHM) and RT-FIFO were adopted to construct the communication between the GUI and real-time tasks. Conclusion: A few experiments of various micro structures micro electrochemical milling on stainless steel are introduced to validate the performance of the system. The process and the results implied that the soft-CNC System is Real-time, stable and reasonable.
\end{abstract}

Key words: Soft computer numerical control, micro Electrochemical Machining (micro-ECM), Graphical User Interface (GUI), Shared Memory (SHM), Block Electrical Discharge Grinding (BEDG), dual-kernel architecture, electrical parameters

\section{INTRODUCTION}

Micro products/systems and components has been widely applied in electronics, optics, medicine, biotechnology, automotive, communications and avionics industries. The production of miniaturized systems/components with micro and sub-micrometer size features require advanced manufacturing technologies to meet the rapidly increasing demand (Qin, 2010). Among the micromachining technologies, micro electrical discharge machining (micro-EDM) and micro electrochemical machining (micro-ECM) offer distinct advantages such as no direct mechanical contact avoids the elastic deformation of work-piece and tool wear or breakage, regardless of the materials mechanical property, can be further extended by combining them or other micromachining methods to form hybrid processes, able to machining 3D micro structures and inexpensive capital investment and have become an important issues in fabrication of small quantities micro-mould or micro-parts (Rajurkar et al., 2006). Compared to EDM, micro-ECM can machine hard metals without mechanical stress, production of heat-affected zone and tool wear and its machining resolution is relatively larger than that of micro-EDM (Chen et al., 2009). Schuster et al. (2000) showed that the machining resolution is limited to a few micrometers by applying ultra short pulses of nanosecond duration and thus, micro structures can be machined by ECM (Schuster et al., 2000; Cagnon et al., 2003). Kim et al. (2005) investigated micro electrochemical milling process, in the research with low concentration electrolyte, $0.1 \mathrm{M} \mathrm{H}_{2} \mathrm{SO}_{4}$, by using of simple cylindrical micro tool electrode, various 3D micro structures with good surface quality were made by layer-by-layer on stainless steel and the ultra-short voltage pulse is also utilized. The pulsed current of nanosecond duration leads to strong spatial confinement of electrochemical reactions down to nanometre precision to achieve high resolution. In addition to the ultra short pulses, a machining process control system and its control policy will evidently affect machining stability and precision. In the process a proper control of the gap results in smaller gap and hence in better shape and dimensional accuracy. Any abnormal status in the small gap that results from poorly performance of the control system or pulse generator, will lead to undesired machining results. Micro-sparks occurring during machining cause uncontrolled material removal and result in improper shape and low accuracy. In order to obtain desired accuracy, a precision machine tool, a credible hardware and software which provide real-time control performance are necessary. In this study a kind of soft computer numerical control (soft-CNC) system based on RT-Linux platform is implemented to meet the real-time control demand for micro-ECM.

Corresponding Author: Yukui Wang, Mechanical Engineering, Harbin Institute of Technology, Harbin, China Fax: 86-0451-86413485 


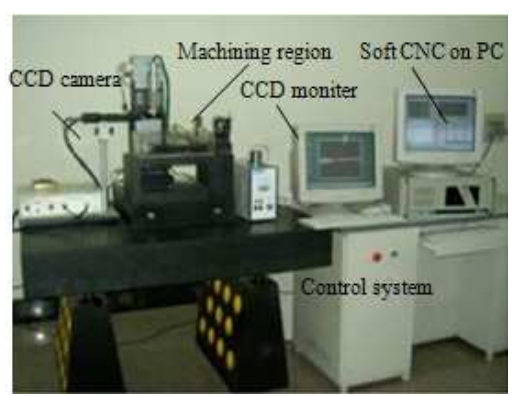

(a)

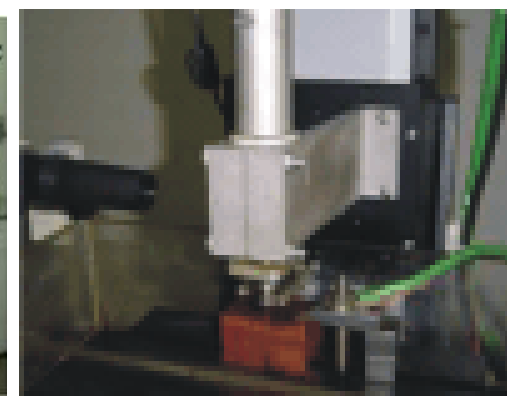

(b)

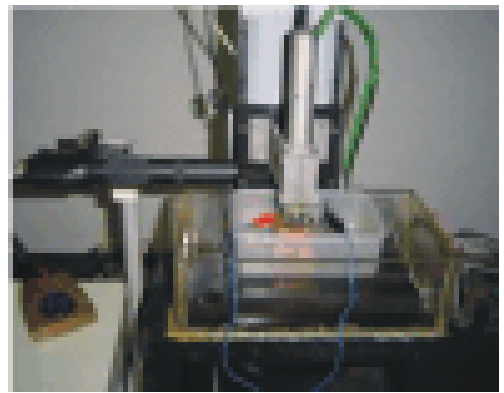

(c)

Fig. 1: Photograph of experimental multifunction machine tool and BEDG/ECM procedure (a) Experimental multifunction machine tool; (b) Electrode by BEDG; (c) ECM procedure

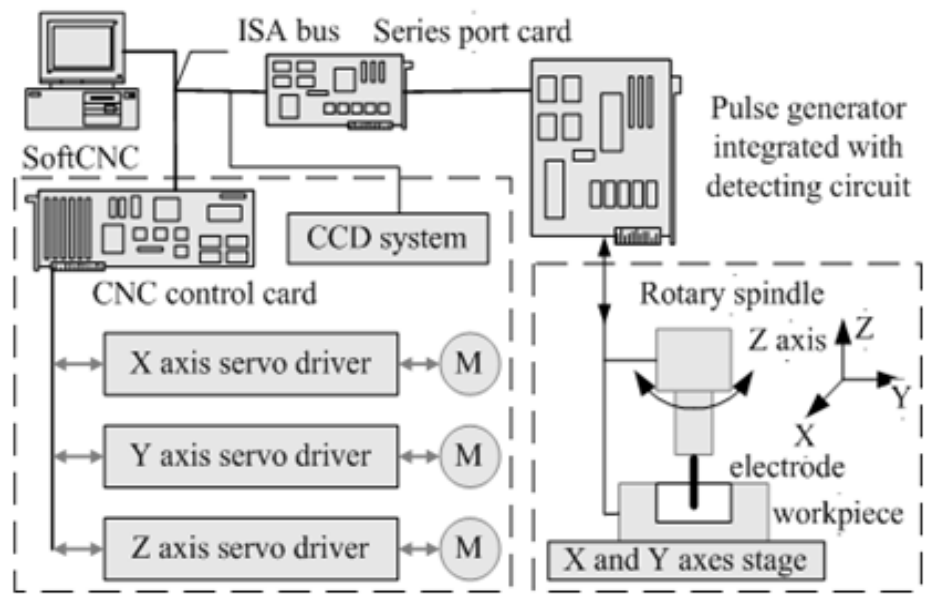

Fig. 2: Diagram of control system for micro-ECM

\section{MATERIALS AND METHODS}

Expérimental setup for micro-ECM: A micro-ECM procedure is introduced on the self-developed multifunction machine tool and their components are described as shown in Fig. 1a. In order to meet the requirements of various micromachining principles, the experimental setup module for microelectrochemical milling is developed independently and expediently for loading or uninstalling on the machine tool. A three-dimensional motion table is carry out by three precise servo devices on granite basement and its journey range is $100 \times 100 \times 100 \mathrm{~mm}$. Every motion component is driven by a precise AC servo motor utilizing high precision grating ruler as position feed-backs so that its resolution of setup reached $0.1 \mu \mathrm{m}$. The precise rotating axis is fixed on the motion components of $\mathrm{Z}$ axis directly and goes up and down with the motion components of $\mathrm{Z}$ axis. The electrode is fixed on the rotating axis which is driven by a DC step motor. The rotating velocity adjusted scope is $0-25000 \mathrm{r} / \mathrm{min}$. The microelectrode can be fabricated online by Block Electrical Discharge Grinding (BEDG) module on the setup as shown in Fig. 1b. In the machine system, both of the motion and electrical parameters must be controlled accurately. An ultra-short pulse generator integrated with detecting circuit is designed. A CNC simple I/O card and a serial port car based on ISA bus are developed for motion and pulse generator control respectively. A computer with soft CNC control software and the PCbased control cards is used to master the whole control functions of the setup including motion numerical control, monitoring of the machining status, regulating of pulse power parameters and acquiring the electrode image by a real-time CCD system. The dimension of electrode can be measured online in the process. The micro-ECM procedure was introduced as shown in Fig. 1c and its control system diagram was described as shown Fig. 2. 
Am. J. Nanotech., 1 (2): 51-55, 2010

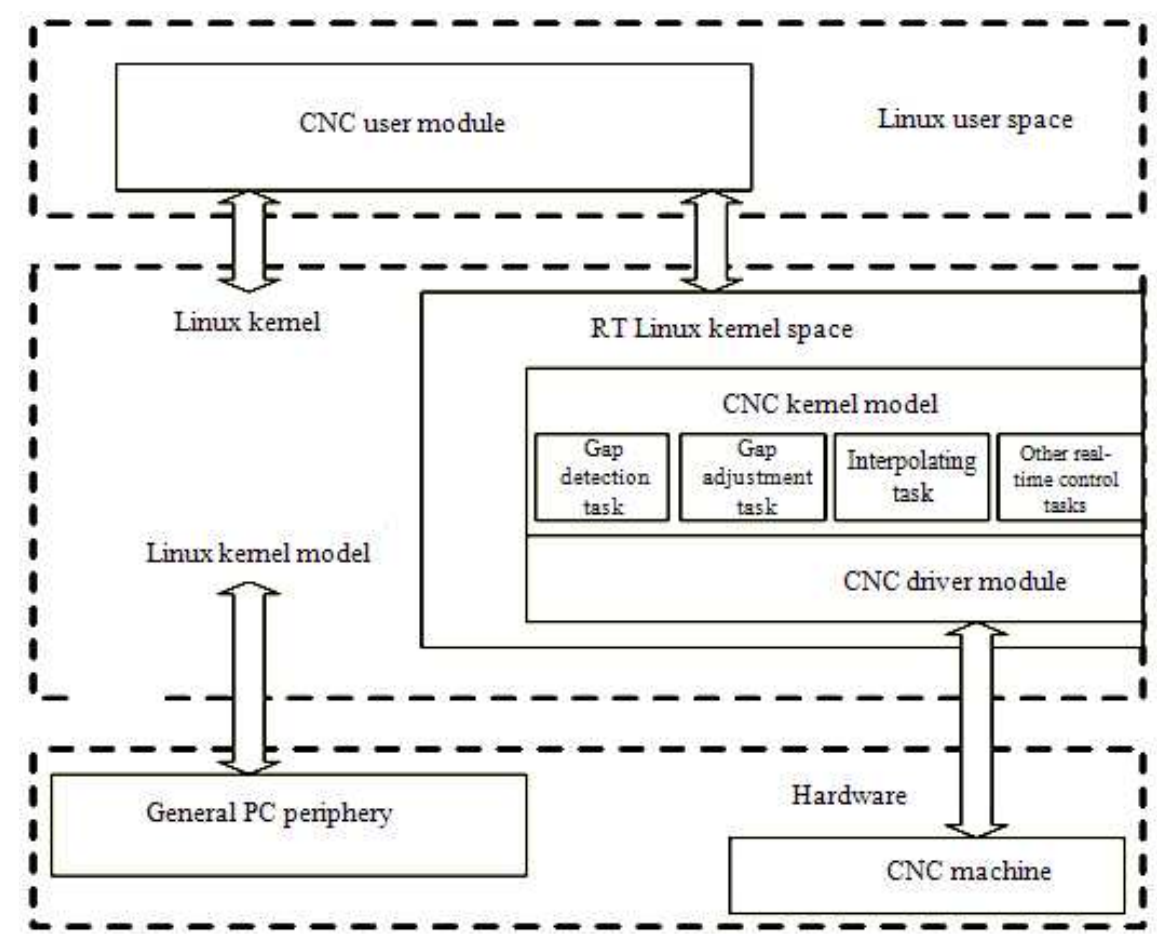

Fig. 3: Soft-CNC structure based on RT-Linux

\section{RESULTS}

\section{Micro-ECM soft-CNC architecture based on RT- Linux:}

RT-Linux: RT-Linux is an operating system in which a small real-time kernel coexists with the Posix-like Linux kernel. The intention is to make use of the sophisticated services and highly optimized average case behaviours of a standard time-shared computer system while still permitting real-time functions to operate in a predictable and low-latency environment. A virtual layer, namely RT-Linux kernel, is added between Linux kernel and hardwares. Using the virtual machine layer for the hardwares, the RT-Linux kernel controls hardwares directly. Linux kernel becomes a thread with minimum PRI of RT-Linux kernel. When RT-Linux kernel receives Real-time task, it executes corresponding Real-time interrupt service routine immediately. When RT-Linux kernel receives Timesharing task, it conserves task message until it is free, then it dispense this task to the Linux kernel. The Realtime task can be responded timely and will not be interrupted by Time-sharing tasks. Considering multitask switching, a task cycle can reach $10 \mu$ s. Recently, it has been applied in lots of occasions there a rigorous
Real-time performance is needed, such as unmanned driving aircraft, open-architecture $\mathrm{CNC}$, rimed turbine blisk machining by EDM and so on (Hai-Peng et al., 2010). Thus the RT-Linux is adopted to develop the CNC system for the micro-ECM process.

Dual-kernel architecture of soft-CNC: The microECM CNC software adopts dual-kernel architecture, as shown in Fig. 3. The whole system was divided into two parts: real-time part and time-sharing part. The real-time part is controlled by RT-Linux kernel and the time-sharing part is controlled by Linux kernel. In periods of switched tasks, the CNC system judges that the applied task is real-time task or time-sharing task at first. If this task is real-time task, it will be taken over by RT-Linux kernel. If this task is time-sharing task, it will be implemented by Linux kernel. The priority of real-time task is higher than that of any time-sharing tasks. When a real-time task arrives, all of the time-sharing tasks are delayed or dormant and have to give up the usufruct of the CPU. Until this real-time task has been accomplished, then other timesharing tasks can go on with their works. By function, the soft-CNC was also classified into three microcosmic modules: $\mathrm{CNC}$ user module, real-time control module and drive module. 


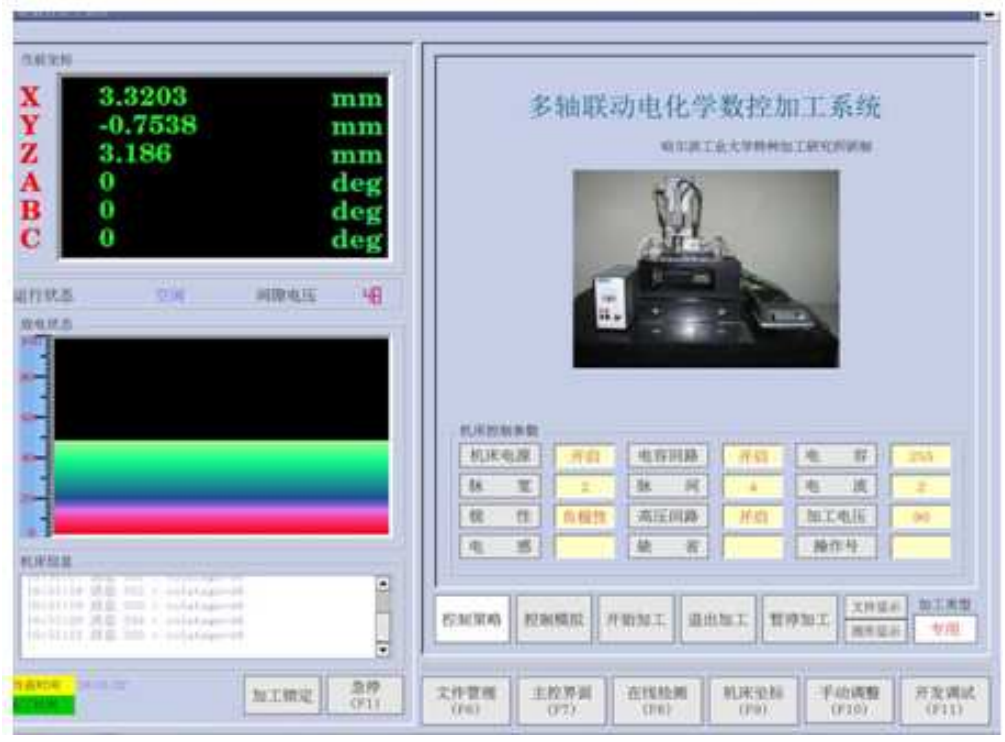

Fig. 4: GUI of the soft-CNC software

CNC user module: $\mathrm{CNC}$ user module which runs on Linux user space, as a manage procedure, was designed by famous GUI tool named Qt and shown in Fig. 4. GUI provides conversation of the machine information, such as coordinates, $\mathrm{G}$ codes, machining status, gap status, the electrical parameters and so on. According to these information manipulator can adjust their machine policies.

Real-time control and driver modules: Based on multi-thread programming, the real-time control module consisting of a gap detection task, a gap adjustment task, an interpolating task and other realtime control tasks was proposed. The module receives the Real-time user commands sent by CNC User Module and puts the commands into Command Buffer. A circular choosing policy is chosen to execute logic of multitask switching. Among these tasks, the gap detection task, gap adjustment task and interpolating task are most important because they execute all the run tasks of the machine. In order to take into account realtime control and multi-task switching, the PRI of gap adjustment task is maximal, the PRI of interpolating task takes second place and the PRI of gap detection task is minimum. In this way, the gap adjustment task is guaranteed to avoid the interference of other tasks and can communicate with other tasks duly when it is necessary, the gap detection task can detect, deposit and renovate the input statuses of special hardwires in real time and wait for the gap adjustment task to get them and the interpolating task responds to the command from the gap adjustment task.
For CNC driver module, there are two kinds of peripheral hardware of EDM CNC system based on RT-Linux. The one is PC general hardware and the other is CNC special hardware. The CNC special hardware is the PC-based cards appending to implement special functions of the CNC machine. Generally, the driver of the PC general hardware is controlled directly by Linux and the driver of CNC special hardware is provided in real-time kernel. CNC driver module is just as device driver level. It is composed of all kinds of drivers of the EDM CNC machine, which mainly include: Driver of servo control card, driver of pulse current source, driver of I/O of the machine and the driver of detection of the machine. The shared memory (SHM) and RT-FIFO were adopted to construct the communication between the GUI, realtime tasks and driver module.

\section{DISCUSSION}

Experiments of micro-ECM: There are micro electrochemical milling experiments of several micro structures introduced on stainless steel 304 to validate the performance of the soft-CNC. In the experiments the $\mathrm{G}$ code for machining complex microstructure was generated by the universal CAD/CAM software UG, electrolyte was low concentration $3 \% \mathrm{NaClO} 3$, machining voltage was $8 \mathrm{~V}$, pulse duration was $200 \mathrm{~ns}$, frequency was $1 \mathrm{MHz}$ and feed rate was $6 \mu \mathrm{m} / \mathrm{min}$. Micro hexagonal star and micro stepped cavity are obtained through the process using rotatory electrodes with 120 and $50 \mu \mathrm{m}$ in diameter separately. 

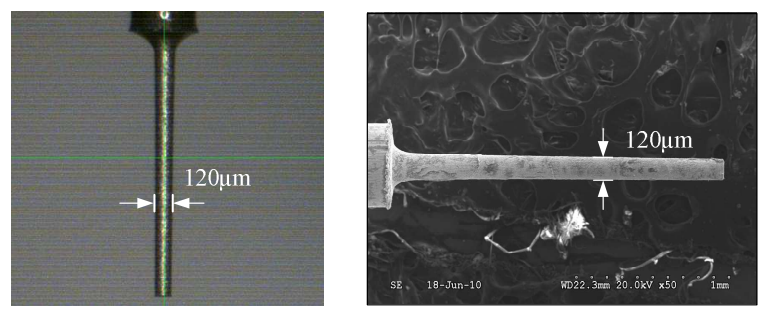

Fig. 5: CCD and SEM photograph of electrode fabricated by BEDG

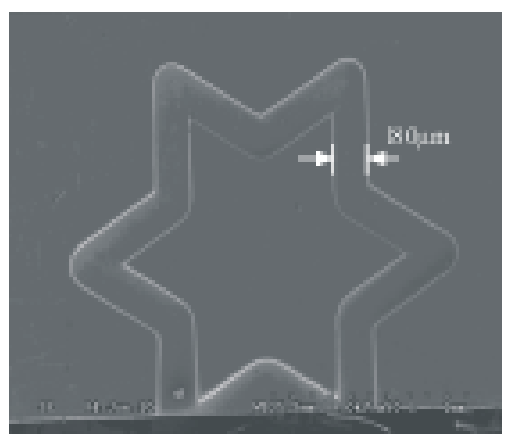

Fig. 6: Micro hexagonal star milled by micro-ECM

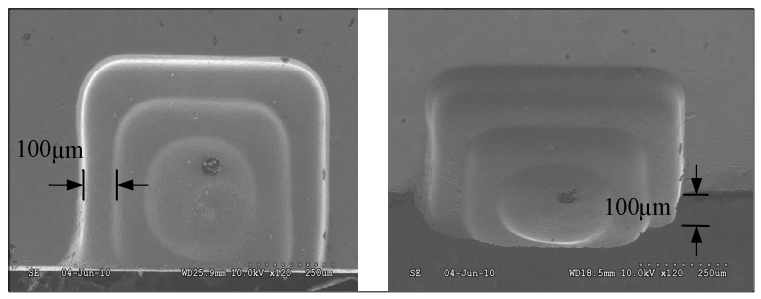

Fig. 7: Micro stepped cavity milled by micro-ECM

The electrode is fabricated online by BEDG and its diameter can be measured online by CCD system. The CCD and SEM photograph of the electrode with $120 \mu \mathrm{m}$ are shown is Fig. 5. In addition to electrode rotation effect, the soft-CNC real-time characteristic keeps the process more stable. Various 3D micro structures with good surface quality were made by layer-by-layer on stainless steel as shown in Fig. 6-7.

\section{CONCLUSION}

In this study a soft-CNC based on RT-Linux for micro-ECM was explored and a few experiments of micro electrochemical milling were introduced to validate soft-CNC performance and stability. The following conclusions are drawn:
- Dual-kernel architecture of soft-CNC for microECM is proposed. RT-Linux and Linux is used to realize Real-time task and Time-sharing task separately

- The real-time tasks consisting of a gap detection task, a gap adjustment task, an interpolating task and other real-time control tasks are designed to take charge of crucial control functions of the softCNC. The CNC graphical user interface (GUI) developed by Qt and running on Linux kernel space provides man-machine conversation function

- The process and the results of electrochemical milling experiment implied that the soft-CNC System is Real-time, stable and reasonable

\section{ACKNOWLEDGMENT}

This research study is funded by Natural Science Foundation in China (No.50835002), Natural Science Foundation of Heilongjiang Province in China (No.E200901) and the Fundamental Research Funds for the Central Universities (No.HIT.NSRIF.2009018).

\section{REFERENCES}

Cagnon, L., V. Kirchner, M. Kock, R. Schuster and G. Ertl et al., 2003. Electrochemical micromachining of stainless steel by ultrashort voltage pulses. Phys. Chem., 217; 299-313. http://link.aip.org/link/doi/10.1063/1.1401783

Chen, H., Z.L. Wang, Z.L. Peng, Y.H. Dong, W.S. Zhao, 2009. Electrochemical micro machining of stainless steel with voltage pulses. Adv. Mater. Res., 69: 219 224. 10.4028/www.scientific.net/AMR.69-70.219

Hai-Peng, H., C. Guan-Xin and W. Zhen-Long, 2010. Multi-axis EDM CNC system based on RT-linux. Key Eng. Mat., 419: 809-812. DOI: 10.4028/www.scientific.net/KEM.419-420.809

Kim, B.H., S.H. Ryu, D.K. Choi and C.N. Chu, 2005. Micro electrochemical milling. J. Micromechan. Microengin., 15: 124-129. DOI: 10.1088/09601317/15/1/019

Qin, Y., 2010. Micromanufacturing Engineering and Technology. Elsevier, pp: 1-23. http://www.lavoisier.fr/livre/notice.asp?id=OSRW AAARAKAOWI

Rajurkar, K.P., G. Levy, A. Malshe, M.M. Sundaram and J. McGeough et al., 2006. Micro and nano machining by electro-physical and chemical processes. CIRP Annal. Manufact. Technol., 55: 643-666. DOI: 10.1016/j.cirp.2006.10.002

Schuster, R., V. Kirchner, P. Allongue and G. Ertl, 2000. Electrochemical micromachining. Science, 289: 98-101. DOI: 10.1126/science.289.5476.98 